\title{
Erratum: Electron cloud instability in high intensity proton rings [Phys. Rev. ST Accel. Beams 5, 114402 (2002)]
}

\author{
K. Ohmi, T. Toyama, and C. Ohmori \\ KEK, 1-1 Oho, Tsukuba, 305-0801, Japan \\ (Received 8 January 2003; published 20 February 2003)
}

DOI: 10.1103/PhysRevSTAB.6.029901

PACS numbers: 29.27.Bd, 29.20.Lq, 99.10.Cd

In the simulation of the electron cloud buildup for SNS, the input parameters were wrong: the beam sizes were different from those given in Table I. The wrong parameter, $\sigma_{r \text {, wrong }}=8.8 \mathrm{~cm}$, was replaced with the correct one, $\sigma_{r}=$ $2.8 \mathrm{~cm}=\sigma_{r \text {,wrong }} / \sqrt{10}$, where $\sqrt{10}$ was due to a wrong value of the emittance 10 times larger. The simulation result for SNS shown in Fig. 2(g) is replaced by the following figure. The results for SNS and PSR are qualitatively consistent with previous studies [1-3]. Our values are a factor of 2 or 3 smaller than those of previous studies. Detailed comparison should be done using the same parameters and conditions.

The corrected values of Tables II and III are displayed in the amended tables. The conclusion for the stability of each machine is corrected as follows: SNS is most severely affected by the electron cloud instability, therefore a cure is required [2,3]. PSR is also severe, while ISIS is safe. J-PARC $3 \mathrm{GeV}$ is in between PSR and ISIS.

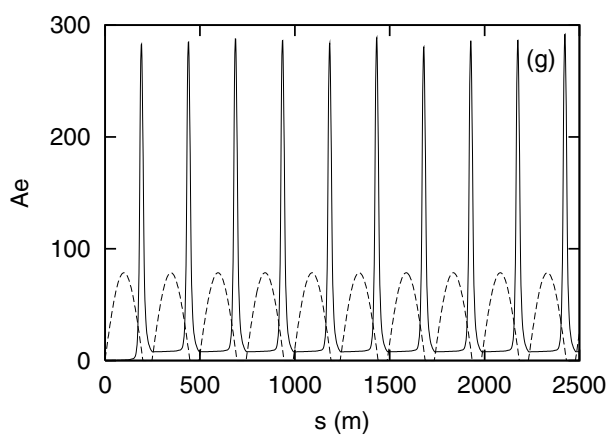

FIG. 2. (g) Electron amplification factor for SNS. The proton beam profiles are plotted with dashed lines in arbitrary units.

TABLE II. Electron cloud buildup of the proton rings.

\begin{tabular}{|c|c|c|c|c|c|c|c|c|}
\hline \multirow[b]{3}{*}{ Variable } & \multicolumn{4}{|c|}{ J-PARC } & \multirow[b]{3}{*}{ PSR } & \multirow[b]{3}{*}{ ISIS } & \multirow[b]{3}{*}{ SNS } & \multirow[b]{3}{*}{ AGS } \\
\hline & \multicolumn{2}{|c|}{$3 \mathrm{GeV}$} & \multicolumn{2}{|c|}{$50 \mathrm{GeV}$} & & & & \\
\hline & Inj. & Ext. & Inj. & Ext. & & & & \\
\hline$A_{e}$ (bottom) & 42.0 & 18.0 & 9.4 & 0.13 & 118 & 12.9 & 7.8 & 0.42 \\
\hline$A_{e}($ peak $)$ & 87.6 & 62 & 136 & 6.9 & 236 & 17.5 & 286 & 5.18 \\
\hline$f$ (bottom) & 0.020 & 0.0067 & 0.0035 & 0.00001 & 0.034 & 0.003 & 0.007 & 0.0001 \\
\hline$f$ (peak) & 0.042 & 0.023 & 0.05 & 0.0005 & 0.067 & 0.005 & 0.25 & 0.0015 \\
\hline
\end{tabular}

TABLE III. Wake field and stability for electron cloud instability.

\begin{tabular}{|c|c|c|c|c|c|c|c|c|}
\hline \multicolumn{9}{|c|}{ J-PARC } \\
\hline & \multicolumn{2}{|c|}{$3 \mathrm{GeV}$} & \multicolumn{2}{|c|}{$50 \mathrm{GeV}$} & \multirow[b]{2}{*}{ PSR } & \multirow[b]{2}{*}{ ISIS } & \multirow[b]{2}{*}{ SNS } & \multirow[b]{2}{*}{ AGS } \\
\hline Variable & Inj. & Ext. & Inj. & Ext. & & & & \\
\hline$Z\left(\omega_{e}\right)_{1, L} / Q(\mathrm{M} \Omega / \mathrm{m})$ & 0.29 & 0.24 & 0.68 & 0.019 & 0.46 & 0.0051 & 0.03 & 0.024 \\
\hline$Z\left(\omega_{e}\right)_{1, H} / Q(\mathrm{M} \Omega / \mathrm{m})$ & 0.61 & 0.83 & 9.7 & 0.96 & 0.90 & 0.0085 & 1.23 & 0.37 \\
\hline$\omega_{e} \widetilde{\ell}_{p} / c$ & 133 & 182 & 199 & 276 & 166 & 27 & 272 & 153 \\
\hline$U_{L}$ & 0.07 & 0.23 & 0.11 & 0.02 & 1.6 & 0.007 & 0.10 & 0.004 \\
\hline$U_{H}$ & 0.15 & 0.78 & 1.6 & 1.2 & 3.2 & 0.012 & 4.0 & 0.06 \\
\hline
\end{tabular}


[1] M. A. Furman and M. Pivi, in Proceedings of the 2001 Particle Accelerator Conference, Chicago, (IEEE, Piscataway, NJ, 2001), p. 707.

[2] M. A. Furman and M. Pivi, in Proceedings of the 2002 European Particle Accelerator Conference, Paris, France (CERN, Geneva, 2002), p. 1547; M. Pivi and M. A. Furman, in Proceedings of the ECLOUDO2 Workshop, CERN, 2002 (CERN Report No. CERN-2002-001, 2002), p. 137.

[3] A. Aleksandrov Danilov, M. Blaskiewicz, and J. Wei, in Proceedings of the 2001 Particle Accelerator Conference, Chicago (Ref. [1]), p. 1749. 